\title{
Desempenho de clones de copa de seringueira resistentes ao mal-das-folhas
}

\author{
Vicente Haroldo de Figueiredo Moraes ${ }^{(1)}$ e Larissa Alexandra Cardoso Moraes $^{(2)}$
}

(1)In memoriam. ${ }^{(2)}$ Embrapa Amazônia Ocidental, Caixa Postal 339, CEP 69011-970 Manaus, AM. E-mail: larissa.moraes@cpaa.embrapa.br

\begin{abstract}
Resumo - O objetivo deste trabalho foi avaliar o desempenho de 18 clones de seringueira resistentes ao Microcyclus ulei, usados como copas enxertadas. Foram utilizados oito clones híbridos de Hevea pauciflora $\mathrm{x}$ Hevea guianensis var. marginata, oito de $H$. pauciflora $\mathrm{x} H$. rigidifolia e dois clones de $H$. pauciflora, enxertados sobre o painel de CNS AM 7905 - seleção primária de H. brasiliensis - e cultivados em Latossolo Amarelo distrófico, em Manaus, AM. Foram avaliados: perímetro do tronco, estado nutricional e produtividade de borracha seca. Copas enxertadas de híbridos $H$. pauciflora x $H$. guianensis var. marginata causam crescimento mais rápido do tronco, com redução do período de imaturidade da seringueira. $\mathrm{O}$ alto nível de resistência dos híbridos de H. rigidifolia ao percevejo-de-renda (Leptopharsa heveae) justifica a introdução de outros genótipos dessa espécie para novas hibridações. Os clones CPAA C 01, 06, 13, 15, 16 e 45 apresentam excelente potencial de produção de borracha seca, nas condições climáticas e de solos de terra firme da Amazônia Tropical Úmida.
\end{abstract}

Termos para indexação: Hevea, Leptopharsa heveae, Microcyclus ulei, aumento do caule, encharcamento do solo, enxertia de copa.

\section{Performance of rubber tree crown clones resistant to South American leaf blight}

\begin{abstract}
The objective of this study was to evaluate the performance of 18 rubber tree clones, resistant to Microcyclus ulei and used as budded crowns. Eight hybrid clones of Hevea pauciflora $\mathrm{x}$ Hevea guianensis var. marginata, eight of $H$. panciflora $\mathrm{x} H$. rigidifolia, and two clones of $H$. pauciflora were used as budded crowns onto CNS AM 7905 - a primary selection of H. brasiliensis - and cultivated in a Xanthic Ferralsol, in Manaus, Amazônia, Brazil. Trunk girth, nutritional status and rubber productivity were evaluated. The budded crowns of hybrids of $H$. pauciflora $\mathrm{x} H$. guianensis var. marginata cause fastest trunk girth increment, with shortening of the imaturity period of rubber tree. The high resistance level of $H$. rigidifolia hybrids to Leptopharsa heveae justifies the introduction of other genotypes of this species, for new hybridizations. The clones CPAA C $01,06,13,15,16$ and 45 present excellent potential for dry rubber production, in the climatic and upland soil conditions of Tropical Amazônia.
\end{abstract}

Index terms: Hevea, Leptopharsa heveae, Microcyclus ulei, girth increments, waterlogged soils, crown budding.

\section{Introdução}

A presença do fungo Microcyclus ulei (P. Henn.) V. Arx., causador do mal-das-folhas da seringueira, tem sido o principal impedimento da expansão da heveicultura na Amazônia Tropical Úmida (Pereira, 1997), exceto em alguns locais dos estados do Mato Grosso e Pará, que apresentam estação de seca prolongada no período da desfolha.

Para viabilizar o cultivo comercial na região, vários estudos foram realizados, mas com resultados pouco animadores. $\mathrm{O}$ melhoramento genético para a obtenção de clones resistentes e produtivos foi ineficaz, por causa da constante quebra da resistência ocasionada pela grande variabilidade e mutabilidade do M. ulei (Sousa \& Moraes, 2001). A substituição das copas susceptíveis por clones de coparesistentes, testados desde 1942, em trabalhos iniciados nas antigas plantações da Ford (Fordlândia) (Pinheiro et al., 1989), com clones de Hevea pauciflora e de Hevea benthamiana, apresentou baixo índice de pegamento, na época, e menor produtividade de borracha seca. Testes em plantios no sul da Bahia, com vários híbridos de copas enxertadas de $H$. brasiliensis x $H$. benthamiana, tiveram a resistência quebrada (Dunham et al., 1982). 
Novas tentativas foram realizadas, como a recuperação de plantios jovens de $H$. brasiliensis, na Amazônia, pela enxertia de copa com clones selecionados de $H$. pauciflora, de resistência estável (Souza \& Lion, 1989). O insucesso, neste caso, se deu não pela infestação do fungo $M$. ulei, mas em razão dos baixos índices de pegamento das enxertias (Souza \& Lion, 1989). Além dos problemas de pegamento, fatores como queda na produtividade de borracha, aliados ao aumento do custo de produção, ocasionado pela adoção da técnica da enxertia, também foram fortes argumentos, na época, contra o uso de clones de copa resistentes ao mal-das-folhas (Bahia \& Sena-Gomes, 1981). Contudo, continuaram os estudos da viabilidade dessa prática de manejo (Moraes, 1995, 2002).

Moraes (1998) relata que o pegamento da enxertia foi satisfatório com o uso de híbridos $H$. pauciflora $\mathrm{x} H$. brasiliensis mas, em plantas adultas, houve incidência severa de danos causados pelo percevejode-renda (Leptopharsa heveae) e de doenças como a queima-do-fio (Kolleroga noxius) e a rubelose (Corticium salmonicolor), bem como a redução do nível de resistência ao mal-das-folhas (M. ulei). Retrocruzamentos com $H$. brasiliensis, do híbrido $H$. brasiliensis $\times$ $H$. benthamiana (copas enxertadas de IAN 6158), foram dizimados por nova estirpe virulenta de M. ulei (Gasparotto et al., 1997), embora tenham anteriormente apresentado resistência parcial a 36 diferentes isolados, o que as havia caracterizado como dotadas de resistência do tipo horizontal.

Tais observações demonstraram a necessidade de se estabelecer novo enfoque na linha do melhoramento genético, especificamente para clones de copa, sob a premissa de que o uso dessa técnica não pode ficar limitado ao emprego dos clones primários de H. pauciflora, ou de híbridos que visem à obtenção de clones resistentes e produtivos.

Após as tentativas com pouco sucesso de seleção de copas de $H$. pauciflora, uma nova linha de pesquisa foi iniciada, com novos cruzamentos, para obtenção de clones de copa de resistência estável ao M. ulei, com alta aptidão ao pegamento da enxertia e efeito depressivo na produtividade reduzido ou nulo (Sousa \& Moraes, 2001). Nessa nova linha, foram escolhidos, para hibridação com $H$. pauciflora, clones de $H$. guianensis var. marginata e H. rigidifolia (ambos com o mesmo grau de resistência estável ao mal-das-folhas) que apresentaram bom pegamento da enxertia.
O objetivo deste trabalho foi avaliar o desempenho de 18 clones de seringueira resistentes ao Microcyclus ulei, usados como copas enxertadas, para cultivo sustentável da seringueira nas condições edafoclimáticas da Amazônia Tropical Úmida.

\section{Material e Métodos}

Foram avaliados 18 clones de copa, em ensaio com sete plantas por parcela e quatro repetições (blocos), no Campo Experimental da Embrapa Amazônia Ocidental, em Manaus, AM ( $3^{\circ} 8^{\prime} 25^{\prime \prime S}$ e 5952'W), em Latossolo Amarelo muito argiloso (730 $\mathrm{g} \mathrm{kg}^{-1}$ de argila), com as seguintes características químicas: $\mathrm{pH}$ (água), 4,3; $\mathrm{N}$ total, $1,55 \mathrm{~g} \mathrm{~kg}^{-1}$; $\mathrm{P}$ (Mehlich 1), $2 \mathrm{mg} \mathrm{dm}^{-3}$; K (Mehlich 1), $47 \mathrm{mg} \mathrm{dm}^{-3} ; \mathrm{Na}$ (Mehlich 1), $7 \mathrm{mg} \mathrm{dm}^{-3} ; \mathrm{Ca}\left(\mathrm{KCl} 1,0 \mathrm{molL}^{-1}\right), 0,24 \mathrm{cmol}_{\mathrm{c}} \mathrm{dm}^{-3}$; $\mathrm{Mg}\left(\mathrm{KCl}\right.$ 1,0 $\left.\mathrm{mol} \mathrm{L}^{-1}\right), 0,12 \mathrm{cmol}_{\mathrm{c}} \mathrm{dm}^{-3} ; \mathrm{Al}\left(\mathrm{KCl} 1,0 \mathrm{~mol} \mathrm{~L}^{-1}\right)$, 1,45 $\mathrm{cmol}_{\mathrm{c}} \mathrm{dm}^{-3} ; \mathrm{H}+\mathrm{Al}, 8,04 \mathrm{cmol}_{\mathrm{c}} \mathrm{dm}^{-3}$; MO (matéria orgânica), 46,89 $\mathrm{g} \mathrm{kg}^{-1}$; $\mathrm{Cu}$ (Mehlich 1), 0,25 mg dm${ }^{-3}$; Fe (Mehlich 1), $333 \mathrm{mg} \mathrm{dm}^{-3}$; Mn (Mehlich 1), 5,15 $\mathrm{mg} \mathrm{dm}^{-3}$ e Zn (Mehlich 1), 0,68 mg dm${ }^{-3} ; \mathrm{V} \%, 6,3$ (Embrapa, 1997).

A área foi desmatada em 1976, com trator de esteira, cultivada com seringueira até 1985, abandonada e novamente destocada com trator de esteira para o preparo do solo. O clima é o tropical úmido, tipo Afi, pela classificação de Köppen, com chuvas relativamente abundantes durante o ano todo (média de $2.250 \mathrm{~mm}$ ), sendo que a quantidade no mês de menor precipitação é sempre superior a $60 \mathrm{~mm}$. A temperatura média anual na região é de aproximadamente $26^{\circ} \mathrm{C}$ (Vieira \& Santos, 1987).

Em canteiros de areia, sementes ilegítimas (cruzamento ao acaso) de $H$. brasiliensis foram colocadas para germinar e, no estágio de crescimento denominado de pata de aranha, foram transplantadas para o viveiro. O transplantio para área definitiva foi feito com toco alto. A enxertia de painel foi realizada com clone CNS AM 7905 (H. brasiliensis, seleção primária em viveiro), e a enxertia com 16 diferentes copas corresponderam aos tratamentos avaliados. O preparo do toco alto com copas enxertadas foi feito conforme as técnicas descritas por Zeid (1977). A enxertia de copa foi realizada aos oito meses, e concluída antes de nove meses após a liberação dos enxertos de base do clone de painel. Foram avaliados oito clones de copa híbridos de $H$. pauciflora x $H$. guianensis var. marginata, oito de $H$. pauciflora $\mathrm{x} H$. rigidifolia, e dois de H. pauciflora, o CPAA C 06 (CNS AM 7745) 
e o CBA 2, utilizados como testemunha. A densidade utilizada foi de 370 plantas por hectare e altura de enxertia de 1,7 m (Moraes, 2002).

O preparo do toco alto para o plantio foi feito de acordo com Moraes (1992) e os tratos culturais foram idênticos aos descritos por Moraes (1995). No preparo da área de plantio definitivo, foram aplicados antes da aração o equivalente a uma tonelada de calcário dolomítico (26,37\% de $\mathrm{CaO}, 12,42 \%$ de $\mathrm{MgO}$ e PRNT de $80 \%$ ) e, dois meses após a calagem, foram aplicados em cobertura: $686,3 \mathrm{~kg} \mathrm{ha}^{-1}$ de superfosfato triplo com $40 \%$ de $\mathrm{P}_{2} \mathrm{O}_{5} ; 5 \mathrm{~kg} \mathrm{ha}^{-1}$ de sulfato de cobre com $13 \%$ de $\mathrm{Cu} ; 5 \mathrm{~kg} \mathrm{ha}^{-1}$ de sulfato de zinco com $20 \%$ de $\mathrm{Zn}$; e $1,5 \mathrm{~kg} \mathrm{ha}^{-1}$ de ácido bórico com $17 \%$ de B. Em seguida, foi realizado o destorroamento do solo com enxada rotativa. Em cobertura, foram aplicados: $696,9 \mathrm{~kg} \mathrm{ha}^{-1}$ de uréia, 464,4 $\mathrm{kg} \mathrm{ha}^{-1}$ de sulfato de amônio e de $696,1 \mathrm{~kg} \mathrm{ha}^{-1}$ cloreto de potássio, fracionados em seis aplicações mensais, a partir do segundo mês.

$\mathrm{Na}$ obtenção dos híbridos interespecíficos, foram utilizados os seguintes clones: $H$. pauciflora: CNS G 112, 118 e 124, CBA 1 e um clone da coleção Baldwin, do antigo IAN, em Belém, PA (H. pauciflora Baldwin); H. guianensis var. marginata: Hgm 1 e Hgm 16; e H. rigidifolia: CNS AM 8105.

Os 16 clones híbridos, provenientes de polinizações controladas, foram obtidos dos seguintes cruzamentos: Hgm 1 x CNS G 112 - CPAA C 01, 13, 14, 15, 18 e 20; CNS G 112 x Hgm 1 - CPAA C 16; Hgm 16 x CBA 1 CPAA C 45; CNS G 118 x CNS AM 8105 - CPAA C 60; CNS AM 7745 x CNS AM 8105 - CPAA C 44, 50, 51 e 52; H. pauciflora Baldwin x CNS AM 8105 - CPAA C 09 e 11; CNS G 124 x CNS NA 8105 CPAA C 33. No primeiro ano, foi realizada seleção preliminar nos clones, de acordo com as características de crescimento, feitas a partir do perímetro do caule, a 1,5 $\mathrm{m}$ do solo. Para calcular os aumentos anuais, foram feitas medições do perímetro do caule a 1,5 $\mathrm{m}$ do solo, em dezembro de cada ano, a partir de 1999 até 2003. Esses dados foram submetidos à análise da variância e as diferenças das médias foram comparadas pelo teste de Tukey, a 5\% de probabilidade (Pimentel-Gomes \& Garcia, 2002).

Inspeções foram feitas a fim de se registrar a ocorrência de pragas e doenças e seleções preliminares foram feitas com base no incremento do diâmetro do caule. No segundo ano após o plantio, foram colhidas amostras de folíolos expostos ao sol, de acordo com
Shorrocks (1962), para avaliação do estado nutricional das plantas. Foram determinados os teores de N, P, $\mathrm{K}, \mathrm{Ca}, \mathrm{Mg}, \mathrm{S}, \mathrm{B}, \mathrm{Cu}, \mathrm{Fe}, \mathrm{Mn}$ e $\mathrm{Zn}$, de acordo com Malavolta et al. (1997).

No quarto ano, foram colhidas amostras de látex, obtidas da sangria sem estimulação, em meio espiral do painel, sob as copas dos clones CPAA C 01, 06 13, 14, 15, 18, 20 e 33, para determinação do teor de magnésio (Moraes \& Moraes, 1997). Em tubo de ensaio com $9 \mathrm{~mL}$ de ácido tricloroacético, a 2,5\%, foi coletada uma amostra composta de $1 \mathrm{~mL}$ de látex, de três plantas por parcela, de cada um dos quatro blocos, aos 10 min após o início da sangria. Os tubos com $10 \%$ de látex foram agitados e colocados em caixa de poliestireno com gelo, até a recepção no laboratório, onde os coágulos foram prensados dentro dos tubos, com bastão de vidro, e então retirados (Moraes \& Moraes, 1995). O teor de magnésio no látex, característica importante na seleção de clones de copa (Moraes \& Moraes, 1997), foi determinado no soro diluído em ácido tricloroacético $2,5 \%$, por absorção atômica, de acordo com Malavolta et al. (1997).

As sangrias das primeiras plantas foram iniciadas em 2004, em plantas com diâmetro superior a $45 \mathrm{~cm}$, sendo feita em meio espiral, com duas sangrias por semana, de acordo com o seguinte modelo: $1 / 2 \mathrm{~s}, \mathrm{~d} / 3$, $6 \mathrm{~d} / 7,12 \mathrm{~m} / \mathrm{y}$, et. $2,5 \%, \mathrm{~Pa}(1: 1), 6 / \mathrm{y}$ (Gonçalves et al., 2001). As produções foram expressas em gramas de borracha seca por árvore por sangria e em quilogramas por hectare ao ano. Nessa fase, foram avaliadas somente as combinações que se mostraram promissoras (CPAA C 01, 06, 13, 15, 16, 45) em comparação à testemunha (CBA 2).

Os resultados de produção de borracha seca também foram submetidos à análise de variância, e as diferenças entre as médias foram comparadas pelo teste de Tukey, a 5\% de probabilidade (Pimentel-Gomes \& Garcia, 2002).

\section{Resultados e Discussão}

$\mathrm{Na}$ seleção preliminar realizada no primeiro ano, foram excluídas as plantas com as copas CPAA C 50, 51 e 52 que apresentaram crescimento muito reduzido nas condições edáficas estudadas, em razão do secamento súbito e morte progressiva das folhas, com posterior emponteiramento das plantas. A elevada incidência desses sintomas, no período de maior intensidade de 
chuvas (dezembro a maio, média mensal de $280 \mathrm{~mm}$ ), e a recuperação parcial que as plantas apresentaram, no período de menor precipitação, evidenciam baixa tolerância desses clones para locais com alto índice pluviométrico ou com predisposição ao encharcamento.

Com exceção dos CPAA C 18, 44, 50 e 51, a percentagem de pegamento das enxertias de copa foi superior a $80 \%$.

Exceto sob a copa CPAA C 20, os perímetros do caule sob os híbridos $H$. pauciflora x $H$. guianensis var. marginata foram significativamente superiores aos dos híbridos $H$. pauciflora $\times$ H. rigidifolia, exceto ao do CPAA C 33 (Tabela 1). O perímetro sob a copa de CPAA C 06 (CNS AM 7745) - um dos clones de H. pauciflora usado como testemunha - não apresentou diferenças significativas em relação aos dos híbridos de $H$. guianensis var. marginata. $\mathrm{O}$ menor perímetro, na média, foi verificado sob a copa da outra testemunha, o clone CBA 2, que não diferiu estatisticamente dos perímetros das copas dos híbridos de $H$. rigidifolia (CPAA C 09, 44 e 60).

Embora o desempenho dos híbridos de $H$. rigidifolia (CPAA C 09, 11, 33, 44 e 60) tenha sido insatisfatório (Tabela 1), a alta resistência ao percevejo-de-renda (Leptopharsa heveae Drake \& Poor) observada - cujo desfolhamento fora da época da troca de folhas do seringal pode reduzir em até $30 \%$ a produção, além de favorecer o ataque do Microcyclus ulei (Gonçalves et al., 2001) - é um caráter valioso que deve continuar sendo explorado, em futuros trabalhos de cruzamento, e justifica a introdução de outros genótipos dessa espécie para novas hibridações. No entanto, também foi verificada a presença de rubelose (Corticium salmonicolor) e crosta-negra (Phyllachora huberi) em plantas isoladas de CPAA C 45 e CPAA C 44, respectivamente, sem danos significativos ao seringal.

Mesmo com as limitações físicas e químicas, apresentadas por grande parte dos solos da Amazônia, predispostos ao encharcamento, o que tem influência negativa no cultivo de culturas anuais e perenes na região (Teixeira, 2001; Moreira \& Fageria, 2008), os aumentos do perímetro do tronco dos clones de seringueira CPAA C 01, 06, 13, 15, 14, 16, 18 e 45 foram altos (Tabela 1), comparados aos de clones de $H$. brasiliensis com copa própria, cujo período de imaturidade estendese, geralmente, por sete anos e, somente em condições excepcionais de clima e solo, a sangria tem início antes dos seis anos (Gonçalves et al., 1993). Sob as copas CPAA C 13, 14, 15 e 18, mais de 50\% das plantas haviam atingido ou ultrapassado o perímetro do caule de $45 \mathrm{~cm}$ (Tabela 1), considerado adequado para o início da realização da sangria (Gonçalves et al., 2001), antes dos cinco anos. Dessas combinações, os clones CPAA C 13 e 14 estavam com $100 \%$ das plantas aptas à sangria nessa idade (Tabela 1).

Nos clones CPAA C 01, 06, 13, 14, 15, 18, 33 e 45, no segundo ano após o plantio, verificou-se que os teores foliares de $\mathrm{N}\left(26 \pm 1 \mathrm{~g} \mathrm{~kg}^{-1}\right), \mathrm{P}\left(1,5 \pm 0,1 \mathrm{~g} \mathrm{~kg}^{-1}\right)$, $\mathrm{Ca}\left(0,4 \pm 0,1 \mathrm{~g} \mathrm{~kg}^{-1}\right)$ e $\mathrm{Mg}\left(1,8 \pm 0,1 \mathrm{~g} \mathrm{~kg}^{-1}\right)$ ficaram

Tabela 1. Avaliações do perímetro do caule $(\mathrm{cm})$ a $1,5 \mathrm{~m}$ de altura e de incremento médio anual (IA, $\mathrm{cm})$, de clones de copa enxertados, em abril de 1999, sobre o painel CNS AM 7905(1).

\begin{tabular}{|c|c|c|c|c|c|c|c|c|c|c|}
\hline \multirow{2}{*}{$\begin{array}{l}\text { Clones de } \\
\text { copa }\end{array}$} & \multirow{2}{*}{$\begin{array}{c}\text { Dezembro } 1999 \\
\text { Perímetro }\end{array}$} & \multicolumn{2}{|c|}{ Dezembro 2000} & \multicolumn{2}{|c|}{ Dezembro 2001} & \multicolumn{2}{|c|}{ Dezembro 2002} & \multicolumn{2}{|c|}{ Dezembro 2003} & \multirow{2}{*}{$\begin{array}{c}\text { Aptos à sangria }{ }^{(2)} \\
(\%)\end{array}$} \\
\hline & & Perímetro & IA & Perímetro & IA & Perímetro & IA & Perímetro & IA & \\
\hline CPAA C 01 & $13,5 \mathrm{ab}$ & $18,0 \mathrm{a}$ & 4,5 & $28,8 \mathrm{ab}$ & 10,8 & $39,3 \mathrm{ab}$ & 10,5 & $47,2 \mathrm{abc}$ & 7,9 & 72,3 \\
\hline CPAA C 06 & $11,2 \mathrm{abcdef}$ & $15,8 \mathrm{abc}$ & 4,6 & $27,4 \mathrm{abcd}$ & 11,6 & $38,0 \mathrm{abc}$ & 10,6 & 45,3 abcde & 8,3 & 79,4 \\
\hline CPAA C 09 & $9,2 \mathrm{ef}$ & $12,3 \mathrm{~cd}$ & 3,1 & $18,9 \mathrm{e}$ & 7,6 & $29,2 \mathrm{de}$ & 11,3 & $37,4 \mathrm{fg}$ & 9,2 & 11,1 \\
\hline CPAA C 11 & $10,0 \mathrm{cdef}$ & $13,7 \mathrm{bcd}$ & 3,7 & 22,9 cde & 9,2 & $34,6 \mathrm{bcd}$ & 11,7 & $40,8 \mathrm{cdef}$ & 6,2 & 30,0 \\
\hline CPAA C 13 & $12,9 \mathrm{abc}$ & $18,1 \mathrm{a}$ & 5,2 & $31,2 \mathrm{a}$ & 13,1 & $43,6 \mathrm{a}$ & 12,4 & $50,6 \mathrm{a}$ & 7,0 & 100,0 \\
\hline CPAA C 14 & $13,6 \mathrm{a}$ & $17,9 \mathrm{a}$ & 4,3 & 27,3abcd & 9,4 & $39,5 \mathrm{ab}$ & 12,2 & $47,8 \mathrm{ab}$ & 8,3 & 100,0 \\
\hline CPAA C 15 & $10,4 \mathrm{cdef}$ & $15,3 \mathrm{abc}$ & 4,9 & 27,5abcd & 12,2 & $39,4 a b$ & 11,9 & $48,6 a b$ & 9,2 & 86,7 \\
\hline CPAA C 16 & $13,9 \mathrm{a}$ & $18,0 \mathrm{a}$ & 4,1 & $28,3 \mathrm{abc}$ & 10,3 & $39,2 \mathrm{ab}$ & 10,9 & 45,3 abcde & 6,1 & 55,0 \\
\hline CPAA C 18 & $13,5 \mathrm{ab}$ & $17,5 \mathrm{a}$ & 4,0 & $28,8 \mathrm{ab}$ & 11,3 & $39,9 \mathrm{ab}$ & 11,1 & $46,8 \mathrm{abcd}$ & 6,9 & 83,3 \\
\hline CPAA C 20 & $12,4 \mathrm{abcd}$ & $16,6 a b$ & 4,2 & $25,2 \mathrm{bcd}$ & 8,6 & $34,6 \mathrm{bcd}$ & 9,4 & $42,4 \mathrm{bcdef}$ & 7,2 & 58,3 \\
\hline CPAA C 33 & $9,5 \mathrm{def}$ & $13,1 \mathrm{bcd}$ & 3,6 & $22,3 \mathrm{de}$ & 9,2 & $34,2 \mathrm{bcd}$ & 11,9 & 44,5abcde & 10,2 & 60,0 \\
\hline CPAA C 44 & 11,4abcde & $15,1 \mathrm{abc}$ & 3,5 & $23,9 \mathrm{bcde}$ & 8,8 & $31,8 \mathrm{cde}$ & 7,1 & 38,6 efg & 6,8 & 0,0 \\
\hline CPAA C 45 & 10,6 bcdef & $15,2 \mathrm{abc}$ & 4,6 & $25,7 \mathrm{abcd}$ & 10,5 & $37,2 \mathrm{bc}$ & 11,5 & 46,8abcd & 9,6 & 66,7 \\
\hline CPAA C 60 & 10,6 bcdef & 14,6abcd & 4,0 & $24,7 \mathrm{bcd}$ & 10,1 & $33,9 \mathrm{bcd}$ & 9,2 & $39,8 \mathrm{defg}$ & 5,9 & 19,4 \\
\hline CBA 2 & $8,4 \mathrm{f}$ & $11,4 d$ & 3,0 & $18,0 \mathrm{e}$ & 6,6 & $27,3 \mathrm{e}$ & 9,3 & $33,5 \mathrm{~g}$ & 6,2 & 16,7 \\
\hline CV $(\%)$ & 11,4 & 9,1 & 12,4 & 13,8 & 10,3 & 12,0 & 15,7 & 12,7 & 13,1 & \\
\hline
\end{tabular}


muito próximos aos indicados como adequados por Pereira \& Pereira (1986), para híbridos H. brasiliensis x $H$. benthamiana, enquanto o $\mathrm{S}\left(2,4 \pm 0,3 \mathrm{~g} \mathrm{~kg}^{-1}\right)$ apresentou teor considerado relativamente alto. Apesar de a seringueira possuir baixa exigência nutricional, as carências de $\mathrm{N}, \mathrm{P}$, $\mathrm{Ca}$ e $\mathrm{Mg}$ podem induzir a formação de casca com menor espessura, menor diâmetro dos vasos laticíferos e menor espessura da casca e do diâmetro do caule (Gonçalves et al., 2001).

Nos clones CPAA C 13, 15 e 33, o teor de $\mathrm{K}$ foi diagnosticado como muito baixo $\left(2,3 \mathrm{~g} \mathrm{~kg}^{-1}\right.$ a $\left.3,5 \mathrm{~g} \mathrm{~kg}^{-1}\right)$, e nos clones CPAA C $01\left(6,5 \mathrm{~g} \mathrm{~kg}^{-1}\right)$ e CPAA C 45 $\left(4,4 \mathrm{~g} \mathrm{~kg}^{-1}\right)$, como baixo (Pereira \& Pereira, 1986), e as plantas apresentaram sintomas típicos da deficiência desse nutriente (Shorrocks, 1964). Já os clones CPAA C 06, $09,11,14,16,18,20,44$ e 60 ficaram dentro da faixa de $9 \mathrm{~g} \mathrm{~kg}^{-1}$ e $12 \mathrm{~g} \mathrm{~kg}^{-1}$, considerada adequada (Pereira \& Pereira, 1986). Os teores médios de $\mathrm{Cu}$ também foram baixos $\left(6 \pm 1 \mathrm{mg} \mathrm{kg}^{-1}\right)$ e os dos demais micronutrientes [B $\left(72 \pm 9 \mathrm{~g} \mathrm{~kg}^{-1}\right), \mathrm{Fe}\left(218 \pm 32 \mathrm{mg} \mathrm{kg}^{-1}\right), \mathrm{Mn}\left(153 \pm 12 \mathrm{mg} \mathrm{kg}^{-1}\right)$ e $\mathrm{Zn}\left(69 \pm 21 \mathrm{mg} \mathrm{kg}^{-1}\right)$ ] situaram-se acima ou dentro dos níveis críticos estabelecidos para H. brasiliensis (Shorrocks, 1962; Guha \& Narayanan, 1969). Na ausência de teores foliares padrões para os clones de híbridos de H. pauciflora e H. guianensis, que apresentam características distintas da H. brasiliensis, optou-se em levar em consideração, além do diagnóstico visual dos sintomas de deficiência, os teores foliares comparados com o padrão $H$. brasiliensis, para definição da necessidade de aplicação adicional de cloreto de potássio e de sulfato de cobre.

No caso dos teores de Mg no látex, foram verificados valores entre 10,54 e 14,12 mM, sob as copas CPAAC 01, $06,13,14,15,20$ e 33. Sob a copa CPAA C 18, o teor de $\mathrm{Mg}$ foi de $6,08 \mathrm{mM}$, o que também indicou a deficiência desse nutriente (Moraes \& Moraes, 1997) e a necessidade de aplicação de $\mathrm{Mg}$ antes do início da sangria, para elevação do teor no látex para valores acima de $10 \mathrm{mM}$, considerado não limitante para a biossíntese da borracha nos laticíferos (Moraes \& Moraes, 1997).

Os resultados de produção de borracha seca, das seis melhores combinações de copa e painel de primeiro ano (2004) e dos nove meses do segundo ano (2005), demonstram bom desempenho quando comparado com a testemunha (CBA 2). Em termos de produtividade por hectare, o clone de copa CPAA C 01 obteve a maior quantidade de borracha seca nos dois primeiros anos de sangria, sendo 118 e $105 \%$, respectivamente, superior à da testemunha (Tabela 2). Tais resultados demonstram
Tabela 2. Resultados das produções do ano de 2004 e a média de nove meses de produção do segundo ano de sangria (2005) dos melhores clones de copa, em comparação com a testemunha (CBA 2). Painel utilizado: CNS AM 7905(1).

\begin{tabular}{|c|c|c|c|c|}
\hline \multirow{2}{*}{$\begin{array}{l}\text { Clone } \\
\text { de copa }\end{array}$} & \multirow{2}{*}{\multicolumn{2}{|c|}{-g por árv. por sangria- }} & \multirow{2}{*}{\multicolumn{2}{|c|}{$2004 \underset{k^{2}}{2005}$}} \\
\hline & & & & \\
\hline$\overline{\text { CPAA C } 01}$ & 27,4 & 36,1 & $975 a$ & $1.284 \mathrm{a}$ \\
\hline CPAA C 06 & 17,2 & 30,6 & $612 \mathrm{~d}$ & $1.088 \mathrm{~b}$ \\
\hline CPAA C 13 & 20,6 & 29,7 & $731 \mathrm{~cd}$ & $1.054 \mathrm{~b}$ \\
\hline CPAA C 15 & 23,3 & 29,1 & $826 b$ & $1.032 \mathrm{~b}$ \\
\hline CPAA C 16 & 21,6 & 27,6 & $767 b c$ & $980 \mathrm{~b}$ \\
\hline CPAA C 45 & 22,1 & 28,4 & $786 b c$ & $1.010 \mathrm{~b}$ \\
\hline CBA 2 & 12,6 & 17,6 & $447 \mathrm{e}$ & $625 c$ \\
\hline
\end{tabular}

(1)Plantio de toco alto com copa enxertada sobre o painel CNS AM 7905; médias seguidas de letras iguais, na coluna, não diferem entre si pelo teste de Tukey, a 5\% de probabilidade.

excelente potencial desse clone, tendo em vista que a produção foi apenas um pouco inferior à observada por Virgens Filho et al. (2003), que avaliaram o clone RRIM 600, de 16 anos, cultivado na região noroeste do Estado de São Paulo, em condições climáticas consideradas aptas para cultivo da cultura por esses autores, mesmo o clone CPAA C 01 estando ainda no início de sangria.

\section{Conclusões}

1. Copas enxertadas de híbridos $H$. pauciflora $\mathrm{x}$ H. guianensis var. marginata conferem crescimento mais rápido do tronco, com redução no período de imaturidade da seringueira.

2. O alto nível de resistência dos híbridos de H. rigidifolia ao percevejo-de-renda, principal praga defolhadora da seringueira, justifica a introdução de outros genótipos dessa espécie para realização de novas hibridações.

3. Há um grande potencial a ser explorado com a determinação de novas combinações copa/painel.

4. Nas condições climáticas e de solos de terra firme da Amazônia Tropical Úmida, as copas de CPAA C 01, $06,13,15,16$ e 45 apresentam excelente potencial de produção de borracha seca.

\section{Referências}

BAHIA, D.B.; SENA-GOMES, A.R. Panel versus crown in some clones of rubber tree (Hevea sp.). Revista Theobroma, v.11, p.203-208, 1981. 
DUNHAM, R.J.; SILVA, E.R.; SANTOS, A.G. Relatório preliminar sobre o desenvolvimento da enxertia de copa da Hevea brasiliensis na Fazenda Três Pancadas. In: SEMINÁRIO SOBRE ENXERTIA DE COPA DE SERINGUEIRA, 1., 1981, Brasília. Anais. Brasília: Sudhevea, 1982. p.92-134.

EMBRAPA. Manual de métodos de análises de solo. Rio de Janeiro: Embrapa-CNPS, 1997. 212p.

GASPAROTTO, L.; SANTOS, A.F. dos; PEREIRA, J.C.R.; FERREIRA, F.A. Doenças da seringueira no Brasil. Brasília: Embrapa-SPI; Manaus: Embrapa-CPAA, 1997. 168p.

GONÇALVES, P. de S.; BATAGLIA, O.C.; ORTOLANI, A.A.; FONSECA, F. da S. Manual de heveicultura para o Estado de São Paulo. Campinas: IAC, 2001. 78p.

GONÇALVES, P. de S.; CARDOSO, M.; MENTE, E.M.; MARTINS, A.L.M.; GOTTARDI, M.V.C.; ORTOLANI, A.A. Desempenho preliminar de clones de seringueira na região de São José do Rio Preto, Planalto do Estado de São Paulo. Bragantia, v.52, p.119-130, 1993.

GUHA, M.M.; NARAYANAN, R. Variation in leaf nutrient content of Hevea with clone and leaf age. Journal of the Rubber Research Institute of Malaya, v.21, p.225-239, 1969.

MALAVOLTA, E.; VITTI, G.C.; OLIVEIRA, S.A. Avaliação do estado nutricional das plantas, princípios e aplicações. Piracicaba: Potafos, 1997. 319p.

MORAES, V.H. de F. Altura da enxertia de copa em seringueira. Agrotrópica, v.14, p.55-60, 2002.

MORAES, V.H. de F. Altura da enxertia de copa em seringueira. Efeitos sobre o crescimento e o custo da enxertia. Manaus: Embrapa-CPAA. 1995. 6p. (Embrapa-CPAA. Pesquisa em Andamento, 18).

MORAES, V.H. de F. Operações de pré-plantio do toco alto de seringueira. Manaus: Embrapa-CPAA, 1992. 40p. (EmbrapaCPAA. Circular Técnica, 5)

MORAES, V.H. de F. Técnicas de enxertia de copa da seringueira. Manaus: Embrapa-CPAA. 1998. 8p.

MORAES, V.H. de F.; MORAES, L.A.C. Diagnóstico do látex em sangria precoce de seringueiras com copas enxertadas: possibilidades de emprego na seleção precoce de clones de copa e de painel. Agrotrópica, v.7, p.49-62, 1995.

MORAES, V.H. de F.; MORAES, L.A.C. Efeito de copas enxertadas de seringueira (Hevea spp.) sobre o teor de magnésio e a regeneração do látex no painel do clone Fx 3899. Agrotrópica, v.9, p.59-66, 1997.
MOREIRA, A.; FAGERIA, N.K. Potential of Brazilian Amazon soil for food and fiber production. Dynamics Soil, Dynamics Plant, v.2, p.82-88, 2008.

PEREIRA, A.V. Avaliação preliminar do desempenho de clones de seringueira (Hevea spp.) no Estado de Goiás e no Distrito Federal. 1997. 98p. Tese (Doutorado) - Universidade Federal de Lavras, Lavras.

PEREIRA, A.V.; PEREIRA, E.B.C. Adubação de seringais de cultivo na Amazônia (Primeira aproximação). Manaus: EmbrapaCNPSD, 1986. 32p. (Embrapa-CNPSD. Circular Técnica, 8).

PIMENTEL-GOMES, F.; GARCIA, C.H. Estatística aplicada a experimentos agronômicos e florestais. Piracicaba: Fealq, 2002. $309 \mathrm{p}$.

PINHEIRO, E.; LIBONATI, V.F.; CASTRO, C.; PINHEIRO, F.S.V. A enxertia de copa na formação de seringais de cultivo nos trópicos úmidos da Amazônia. In: EMBRAPA. Enxertia de copa da Seringueira. Manaus: Sudhevea, 1989. p.63-81. (EmbrapaCNPSD. Documentos, 7).

SHORROCKS, V.M. Leaf analysis as a guide to nutrition of Hevea brasiliensis. V. Leaf sampling technique for mature trees. Journal of the Rubber Research Institute of Malaya, v.17, p.167-190, 1962.

SHORROCKS, V.M. Mineral deficiencies in Hevea and associated cover plants. Birmigham: Kyoch Press, 1964. 76p.

SOUSA, N.R.; MORAES, V.H.F. Recursos genéticos de Hevea. In: SOUSA, N.R.; SOUZA, A. das G.C. (Ed.). Recursos fitogenéticos na Amazônia Ocidental; conservação, pesquisa e utilização. Manaus: Embrapa Amazônia Ocidental, 2001. p.189-199.

SOUZA, M.I.R.; LION, A. Observações de campo na Guamá Agro-Industrial (Grupo Pirelli). In: EMBRAPA. Enxertia de copa da Seringueira. Manaus: Sudhevea, 1989. p.21-27.

TEIXEIRA, W.G. Land use effects on soil physical and hydraulic properties of a clayey Ferralsol in the Central Amazonia. Bayreuther: Bodenkundlicher Berichte. 2001. 255p.

VIEIRA, L.S.; SANTOS, P.C.T.C. dos. Amazônia: seus solos e outros recursos naturais. São Paulo: Agronômica Ceres, 1987. 416p.

VIRGENS FILHO, A. de C.; MOREIRA, A.; CASTRO, P.R. de C. e. Efeitos da adubação NPK e da calagem na fertilidade do solo e na produção da seringueira no Estado de São Paulo. Agrotrópica, v.15, p.61-68, 2003.

ZEID, P. Interim report on the development of advanced planting material. In: RUBBER RESEARCH INSTITUTE OF MALAYSIA PLANTER'S CONFERENCE, 1., 1977, Kuala Lumpur. Proceedings. Kuala Lumpur: RRIM, 1977. p.21-46.

Recebido em 15 de julho de 2008 e aprovado em 17 de outubro de 2008 